\title{
El rol del Marketing Estratégico en las Organizaciones inmersas en entornos VUCA
}

\author{
The Role of Strategic Marketing in Organizations immersed in VUCA environments
}

\author{
Margarita Ramírez-Torres* \\ Universidad Autónoma de Baja California - México \\ ORCID iD: https://orcid.org/0000-0002-7628-6442 \\ mramirez@uabc.edu.mx

\section{Erick P. Salinas-Chaidez} \\ Universidad Autónoma de Baja California - México \\ ORCID iD: https://orcid.org/0000-0002-5138-5233 \\ paul.salunas@uabc.edu.mx
}

\section{Ana B. Miranda-Navarro \\ Universidad Autónoma de Baja California - México \\ ORCID iD: https://orcid.org/0000-0002-6759-8567 \\ ana.miranda@uabc.edu.mx}

* Autor a quien debe ser dirigida la correspondencia
Fecha de recepción: 22/09/2021

Fecha de evaluación: 19/10/2021

Fecha de aceptación: 01/11/2021

Cómo citar: Ramírez-Torres, M., Salinas-Chaidez, E., \& Miranda-Navarro, A. (2021). El rol del Marketing Estratégico en las Organizaciones inmersas en entornos VUCA. Revista Científica Anfibios, 4(2), 45-54. https://doi.org/10.37979/afb.2021v4n2.94

\section{Resumen}

Aspectos como el cambio en el consumo de los clientes, el aumento de éstos y sus demandas específicas y las nuevas tecnologías están provocando un entorno donde la volatilidad, la incertidumbre, la complejidad y la ambigüedad resulta habitual para las organizaciones. En ese marco, se plantea un análisis acerca del manejo del marketing estratégico de las organizaciones haciendo énfasis en la importancia de la estrategia para la gestión de las empresas, así como la importancia de la medición del rendimiento organizacional y su eficiencia. Para tales fines se plantea un análisis de la literatura enmarcando los principales aspectos que vinculan estas temáticas. Los resultados apuntan a que el marketing estratégico tiene una influencia positiva en los resultados de la empresa, lo que se traduce en una operación eficiente y eficaz, sólida, además de ser rentable a largo plazo. Un buen plan estratégico es fundamental para la consecución de los objetivos comerciales de la compañía y su permanencia en el mercado.

\section{Palabras clave}

Marketing estratégico; organizaciones; clientes; VUCA

\begin{abstract}
Aspects such as the change in customer consumption, the increase in the number of customers and new technologies are causing an environment where volatility, uncertainty, complexity and ambiguity are common for organizations. Within this framework, an analysis of the strategic marketing management of organizations is proposed, emphasizing the importance of strategy for the management of companies, as well as the importance of measuring organizational performance and its efficiency. For such purposes, an analysis of the literature is proposed, framing the main aspects that link these topics. The results show that strategic marketing has a positive influence on the company's results, which translates into an efficient, effective and solid operation, in addition to being profitable in the long term. A good strategic plan is fundamental for the achievement of the company's business objectives and its permanence in the market.
\end{abstract}

\section{Keywords}

Strategic marketing; organizations; customers; VUCA 


\section{Introducción}

Actualmente las empresas están en constante competencia con el fin de posicionarse en el gusto del consumidor ofreciendo una ventaja competitiva a largo plazo(Pacheco \& León Sinche, 2017; Srinivasan \& Lohith, 2017), pero no todas las empresas consiguen eso, es importante que se utilice o se implementen estrategias efectivas de mercadotecnia para cumplir con el fin (Tokman et al., 2019).

Los esfuerzos del marketing en las empresas han sido herramientas funcionales que impactan favorablemente, las buenas prácticas que se han desarrollado es el punto que marca la diferencia (Reddy \& Reinartz, 2017). Según Kotler \& Keller, (2006) para una correcta planificación estratégica empresarial, la empresa debe definir su misión respondiendo a determinadas preguntas asociadas con el negocio, los clientes y su valor agregado.

El posicionamiento es indispensable en una sociedad que se encuentra saturada de información; esta es la percepción que los consumidores tienen sobre ciertas marcas o empresas en relación a sus competidores (Minciu et al., 2020). En ese sentido, hoy en día es necesario que las empresas empiecen a tener un panorama integral de la organización, para que no solo se concentren en la parte de hacer rentable el negocio, sino que se aproveche todo el potencial que la empresa tiene para generar utilidad, bienestar, crecimiento propio y desarrollo social (Torres Huaman, 2020). Esto es fundamental en el mundo empresarial actual caracterizado por la volatilidad y la inestabilidad, por lo que la anticipación estratégica resulta en una herramienta indispensable (Del Rio Cortina, 2021)

Para Fraj et al., (2015) hay diferentes posturas con respecto de las teorías que discuten el rendimiento y su relación con la práctica. En lo particular la teoría basada en recursos se me hace apropiada, ya que sostiene que la ventaja competitiva y el rendimiento dependen de las dotaciones de recursos desarrollados a través del tiempo(Gaytán Ramírez \& Flores Villanueva, 2018). Como resultado los negocios deben concentrarse en los recursos que les generen capacidad de producción de bienes de valor, poco imitables y que sea de forma eficiente y efectiva(Martínez Santa María et al., 2010; Mullins et al., 2019).
El aprendizaje y conocimiento de los clientes y el mercado, constituyen elementos muy difíciles de duplicar, son una base sólida para el marketing y para poder desarrollar una posición dentro del mercado (Atuahene-Gima \& Murray, 2004; Quist et al., 2011; Ramanathan et al., 2016). Estos recursos le dan a la empresa una posibilidad de poseer una competencia distintiva, que pueden sumar a la valoración de la empresa en un futuro (Bao, 2015).

Es así que se propone analizar el rol del Marketing Estratégico en las Organizaciones inmersas en entornos VUCA con la finalidad de estudiar los principales hallazgos teóricos y prácticos en este tema, es por esto que se inicia con los referentes teóricos estableciendo los principales referentes en la temática del marketing estratégico, posteriormente, se anuncian los aspectos metodológicos relacionados con una revisión de la literatura para anunciar los principales resultados y conclusiones.

\section{Referentes Teóricos}

La mercadotecnia es entendida en términos generales como El proceso por el cual una organización participa en el mercado de manera creativa, productiva y rentable (Halim et al., 2019; Hanggraeni et al., 2019; Moliner-Velázquez et al., 2019; Tokman et al., 2019), en otras palabra, es el arte de generar y satisfacer necesidades de los clientes obteniendo una utilidad (Ali et al., 2018; Marcos Cuevas, 2018). Todas las empresas están sujetas a muchas variables, algunas controlables y otras que se encuentran fuera de su alcance, para eso, se han englobado en cuatro factores principales para el cumplimiento de los objetivos según los valores de las instituciones: producto, precio, plaza y promoción(Agostini et al., 2015; Asare et al., 2015; Franco Restrepo et al., 2014).

Por otra parte, es de suma importancia aplicar la planificación estratégica cuando se quiera redefinir o direccionar algunos procesos para la dirección y toma de decisiones sobre las metas a perseguir, es la guía que se tiene previamente para modificar los objetivos preestablecidos (Rey \& Bastons, 2018; Saritas \& Kuzminov, 2017)

Sin duda la práctica y experiencia que se va adquiriendo en el modo del proceso, así como las estrategias que se van aplicando cuando surge 
momentos inesperados ha posicionado al país a nivel mundial, creando variados beneficios al país (Bolisani \& Bratianu, 2017; Keatlholetswe $\&$ Malete, 2019). Para Tomas (2021) una estrategia de marketing es el proceso que permite que una empresa se centre en los recursos disponibles y los utilice de la mejor manera posible para incrementar las ventas y obtener ventajas respecto de la competencia (Abeles et al., 2017; Burbano et al., 2019).

La antes mencionada definición de estrategia de marketing, se alinea con la teoría basada en recursos, ya que la empresa se enfoca en los recursos de los que dispone y con ello se encarga de incrementar las ventas y obtener ventaja sobre su competencia, teniendo en cuenta que las estrategias de mercadotécnica surgen de los objetivos de la empresa(Fraj et al., 2015; Kowalkowski et al., 2017). Por lo tanto, es deber de la mercadotecnia brindar las herramientas necesarias para que la empresa logre ingresos y se consolide con un crecimiento rentable y sostenido (Castro Monge, 2010)

Según Ruiz (2021), es necesario evaluar los resultados de la estrategia comercial. Realizar la relación entre las ganancias obtenidas y los esfuerzos que se invirtieron en el proceso. Pero el mejorar la rentabilidad no solo es el aumento de las ventas, ya que lo anterior puede implicar un aumento en los recursos requeridos, que como resultado podría disminuir la rentabilidad comercial (Del Río-Cortina et al., 2017)

El éxito de un negocio no se trata solo de vender más, sino de generar utilidades. Para evaluar los resultados y conocer si la inversión es rentable, se debe establecer objetivos inteligentes y utilizar métricas que evalúen su cumplimiento. Los indicadores clave de rendimiento o KPI's (Key Performance Indicator por sus siglas en inglés), son métricas que pueden ayudar a calcular el rendimiento de la empresa, pues, miden el estado, efectividad y progreso de los objetivos (Milichovsky \& Simberova, 2015; Mizik, 2014; Mizik \& Jacobson, 2008)

Es necesario que la organización analice de forma detenida el entorno en que opera, para que esto pueda significar a largo plazo, una operación exitosa, aprovechando nuestras fortalezas y contemplando las debilidades de la competencia. Según Chirico \& Salvato, (2008) una empresa debe de ser lo suficientemente sólida para competir con empresas de su mismo giro dentro de la industria local o interna, tanto como dentro de la industria internacional o externa. Una empresa para consolidarse a largo plazo, necesita hacer un análisis de su entorno externo, muy particular de sus clientes, competencia, consumidores, proveedores, acreedores y gobierno, identificar la forma en que afectan sus operaciones, teniendo en cuenta la productividad, satisfacción del cliente y la fortaleza de su competencia (Fraj et al., 2015; Masoom \& Anwar, 2013; Park et al., 2010).

Moliner-Velázquez et al., (2019) sugieren que el proceso de desarrollo de prácticas estratégicas de marketing ayuda a garantizar que todos los programas de marketing táctico apoyen las metas y objetivos de la empresa, así como transmitir un mensaje coherente a los clientes. Este enfoque mejora la eficiencia de la empresa en todas las áreas, lo que ayuda a mejorar los ingresos y el crecimiento de la cuota de mercado, y minimiza los gastos, todo lo cual conduce a una mayor rentabilidad. Con ello es importante tener en cuenta la opinión del cliente, cuáles son sus necesidades actuales que están sustentando sus compras, o cuáles son sus motivaciones al iniciar su proceso de compra, tendencias que modifiquen estas conductas, a favor de la compañía o a favor de la competencia, ofreciendo productos similares a los propios (Buil et al., 2010; Romaniuk et al., 2017).

Para construir marcas fuertes, se necesitan estrategias de marketing bien planificadas y bien ejecutadas. Para tener éxito, las empresas también tienen que estudiar cuidadosa y continuamente al consumidor, tendencias de comportamiento a lo largo del tiempo y ajustar en consecuencia (Fombrun \& Gardberg, 2000; Shih, 2018). En este sentido podemos afirmar que se encuentra que el marketing estratégico está relacionado de forma positiva con el rendimiento.

Es prudente añadir que los recursos y estrategias, aunque sean idénticos, no pueden obtener los mismos resultados, debido a las diferencias de la cultura y las preferencias de los consumidores. Cualquiera que sea el entorno, el trabajo del departamento de marketing es adaptar la estrategia de una empresa a las diferentes condiciones ambientales de una manera que produzca una respuesta favorable (Clark, 2000). 
De acuerdo con Ruíz (2021), la rentabilidad comercial, desde una perspectiva financiera, se define como un ratio que evalúa la calidad comercial de una empresa y es el resultado de la división de los beneficios (ganancias) obtenidos de las ventas entre los esfuerzos (inversión) hechos en determinado periodo de tiempo. Asimismo, utilizar este ratio para evaluar tu estrategia comercial puede ayudarte a esclarecer las acciones que deben seguir tus equipos de marketing y ventas.

Una empresa debe de considerar en la actualidad ser parte del mundo digital, ya que no importa el giro al que pertenece, el expandir sus canales de venta y distribución le dará una ventaja, además que con las condiciones actuales se ha vuelto indispensable en el día a día. Según Ruíz (2021) debido a la competitividad que existe en este mundo digital, las empresas pelean por conseguir más clientes y reducir sus costos al mismo tiempo. Tener una empresa no es suficiente si no estás implementando acciones que mejoren su rendimiento. Para lograrlo, es necesario trazar un plan estratégico que te ayude a incrementar las ventas y reducir gastos. El mercado cambia constantemente y las acciones que funcionaron ayer, pueden no servir hoy. Impulsar la eficiencia operativa de los departamentos de marketing y ventas, lo que conduce a generar un mejor ROI.

Es muy importante establecer metas claras. Para alcanzarlas e incluso superarlas, es indispensable unificar los esfuerzos de los equipos. Es vital establecer objetivos para reducir errores de comunicación y ejecución, generando una mejor retroalimentación entre toda la organización. El crecimiento de una empresa es la consecuencia de establecer una buena dirección con metas claras y concisas, así como, la mejora continua de las acciones para alcanzar sus objetivos. Los objetivos deben ser específicos, medibles, alcanzables, relevantes y en un tiempo determinado (objetivos SMART, por sus siglas en inglés). (Ruíz, 2021).

\section{Metodología}

Basado en un enfoque cualitativo y enmarcado en una metodología descriptiva y analítica, se planteó de revisión de la literatura a partir fuentes bibliográficas con referencias nacionales e internacionales (Hoyos \& Sastoque, 2020) buscando analizar el papel del marketing estratégico en las organizaciones inmersas en entornos VUCA. De forma específica, se buscaron palabras claves en plataformas científicas como Web of Science y Scopus, con la finalidad de obtener los aportes mas representativos de la literatura científica, en ese sentido, el criterio de inclusión estuvo vinculado al grado de contribución, así como el sustento empírico de los resultados. Posteriormente, se analizaron los principales hallazgos y conclusiones construyendo los resultados planteados.

\section{Resultados}

Es una realidad que todas las naciones se encuentran interconectadas con otras, de manera económica, política, social y tecnológica. En décadas anteriores, un producto o servicio podría ser no tan popular, difícil de encontrar o simplemente no existir(Minciu et al., 2020).

Actualmente, es posible adquirir cualquier producto o servicio de manera fácil y rápida, gracias a las compras en línea que pueden hacerse a través de las redes sociales y diversas plataformas en internet; esto como parte de las estrategias y tácticas implementadas por las empresas que ofertan diversos productos y servicios a través de la red (Peltier et al., 2020)

Cabe mencionar que esta facilidad de compraventa de productos y servicios en la actualidad, es algo que incrementa la necesidad de competitividad de las empresas. Debido a esto, la implementación de estrategias de marketing adecuadas y personalizadas son importantes para alcanzar los objetivos de la organización (Prawira, 2019).

La planificación estratégica de mercado marca la dirección que debe seguir una empresa y juega un papel fundamental en la consecución de los objetivos de largo plazo de crecimiento de las ventas, beneficios y cuota de mercado. (Best, 2007)

Para hablar del marketing estratégico, debemos mencionar también lo que significan los conceptos de estrategia y táctica dentro del marketing. Podemos entender a la estrategia como el camino que se crea para alcanzar los objetivos o metas específicas de una empresa, mientras que, por otro lado, la táctica consiste en explicar a detalle y/o desarrollar dicha estrategia. Dicho esto, podemos concluir que una depende de la otra. 
Para que las estrategias funcionen, debe conocerse el entorno de la compañía, es decir, cuáles son sus fortalezas y debilidades al interior y al exterior. El marketing estratégico debe entonces, evaluar la situación actual de la empresa a través de la implementación de un análisis FODA para crear las estrategias adecuadas a fin de alcanzar los objetivos planteados por la empresa.

Para que una empresa pueda alcanzar el éxito deseado, se debe analizar a profundidad la situación en la que se encuentra, sin embargo, me parece que es indispensable evaluar las necesidades del mercado a quien va dirigido nuestro servicio o producto, ya que actualmente el hecho de tener una grandiosa y novedosa idea no es sinónimo de que la empresa prospere.

Algo que podríamos tomar en cuenta para posicionarnos en el mercado, es trabajar en la diferenciación de marca. Para esto, hay que hacer un trabajo hacia el interior para que este se note en el exterior. Actualmente, trabajamos con intangibilidades como las percepciones y la reputación. Sería interesante conocer en qué nivel se encuentra la industria floral en Kenia respecto al resto de la competencia.

Por otro lado, ¿cómo saber que nuestra organización está teniendo el éxito esperado? Existe una forma de conocer si estamos por alcanzar nuestros objetivos y es a través de la medición del desempeño organizacional. Al medirlo, podemos tener una idea de que tan bien le está yendo sobre el alcance de los objetivos y metas trazados.

La finalidad de medir el desempeño organizacional es aumentar la efectividad y eficiencia de la organización sobre la venta de los bienes o prestación de servicios.

Cabe mencionar que eficiencia se refiere a "hacer bien las cosas", es decir, al cómo se hacen las cosas; en este caso, dentro de una organización. Esto reúne entonces los factores de personal, procesos y clientes. Una empresa es eficiente cuando el personal que labora en la empresa da un servicio de calidad por medio de procesos fluidos que ofrecen al cliente una experiencia agradable.

Una mayor valorización inmaterial del actuar de la empresa, sus servicios y valores se convierten en factores de diferenciación más relevantes para la sociedad global, regional y local. La trans- formación de la cultura del dominio del poder de transacción hacia una cultura de la atención y del estar "al servicio" implica atender y dar solución a las necesidades de los clientes y de los steakholders. (Garrido \& Putnam, 2018, p.32)

Lo anterior es fundamental, ya que nos permite analizar los resultados, medir su impacto y con ello poder identificar áreas de oportunidad, poder realizar un análisis del trabajo del equipo comercial, permitirnos realizar una proyección de ingresos y con ello prevenir errores que pudieran cometerse.

Para que una empresa sea eficiente y eficaz, no basta con ser solamente rentable. Tiene que establecer estrategias y elaborar un plan de estratégico de mercadotecnia, que constituya las base que guíe a la consecución de las mismas; lo que se traducirá en una empresa sólida, con capacidad de enfrentar los cambios que se originen en el mercado y además con la fuerza para responder a las acciones que tome la competencia.

Es importante que la empresa cuente con mecanismos que le ayuden a medir qué tanto está avanzando, para que pueda con ello capitalizar los esfuerzos que está invirtiendo en acciones, que a corto, mediano o largo plazo lo ayuden a llegar a su objetivo planteado. Adaptarse a los cambios, innovar y seguir avanzando. Por último y no menos importante, escuchar las necesidades de sus clientes, adecuar las mejores prácticas de empresas nacionales e internacionales a su entorno y a las características del mercado.

\section{Conclusiones}

Como lo mencionan Garrido \& Putman (2018) la estrategia no puede ser copiada de otro lado, debido a que el contexto, características internas y externas de la compañía no son iguales a las de otras compañías o empresas. Es absurdo querer transplantarlo ya que su performance será diferente, por ende, los resultados podrán ser muy distintos.

En ese sentido, vivimos en un mundo globalizado donde el ser competitivo es lo que te hace sobresalir, y en cuestión de negocios esto ha quedado claro ya que en la actualidad se buscan continuamente mejores estrategias para la productividad que permitan llegar a los objetivos planteados por la organización. 
Los factores internos y externos se deberán considerar a la hora de tomar decisiones sobre qué estrategias de marketing se van a llevar a cabo dentro de las empresas.

Podemos definir que la competencia en las empresas, sin importar los sectores está en el estudio y análisis de ver las necesidades de los consumidores y la búsqueda constante de satisfacerlas, implementando estrategias para alcanzar los objetivos predeterminados, pero sobre todo crear una oferta de valor que pueda ser el diferenciador de la competencia, así como plantear un modelo de gestión que guíe el actuar de la empresa.

Por citar un ejemplo de caso de éxito está la empresa Southwest Airlines, que hoy en día es imitada por las grandes empresas a nivel mundial ya que redefinió el modo de vuelo, con precios bajos, menos tiempo de espera en tierra, colaboradores de valor (excelente servicio al cliente), rutas cortas y frecuentes; Su modelo tiene tres componentes básicos: el producto, la estructura y las relaciones, esto es un claro ejemplo de un proceso que ha posicionado a la aerolínea como una de las mejores (Gittell, 2003)

Finalmente, el marketing estratégico es indispensable para la supervivencia de las empresas y organizaciones en la actualidad, pues básicamente lo que se busca es la innovación e implementación de estrategias y tácticas que logren la prosperidad y posicionamiento de las mismas; sin embargo, es importante recalcar que una estrategia no puede ser "calcada", ya que estas son únicas puesto que las organizaciones son sistemas vivos e irrepetibles.

\section{Referencias}

Abeles, M., Cimoli, M., \& Lavarello, P. (2017). Manufactura y cambio estructural. Aportes para pensar la política industrial en la Argentina. In Desarrollo Económico. https://repositorio.cepal.org//handle/11362/42393\%0Ahttps:/www.cepal.org/es/publicaciones/42393-manufactura-cambio-estructural-aportes-pensar-la-politica-industrial-la

Agostini, L., Filippini, R., \& Nosella, A. (2015). Brand-Building Efforts and Their Association with SME Sales Performance. Journal of Small Business Management, 53(S1), 161-173. https:// doi.org/10.1111/jsbm.12185

Ali, A., Xiaoling, G., Sherwani, M., \& Ali, A. (2018). Antecedents of consumers' Halal brand purchase intention: an integrated approach. Management Decision, 56(4), 715-735. https://doi. org/10.1108/MD-11-2016-0785

Asare, R., Akuffobea, M., Quaye, W., \& Atta-Antwi, K. (2015). Characteristics of micro, small and medium enterprises in Ghana: Gender and implications for economic growth. African Journal of Science, Technology, Innovation and Development, 7(1), 26-35. https://doi.org/10.1080/2 0421338.2014.979651

Atuahene-Gima, K., \& Murray, J. Y. (2004). Antecedents and Outcomes of Marketing Strategy Comprehensiveness. Journal of Marketing, 68(4), 33-46. https://doi.org/10.1509/jmkg.68.4.33.42732

Bao, G. (2015). What theories are needed for strategic management? Nankai Business Review International, 6(4), 433-454. https://doi.org/10.1108/NBRI-05-2015-0012

Best, R.J. (2007) Marketing Estratégico. Pearson Education. Madrid.

Bolisani, E., \& Bratianu, C. (2017). Knowledge strategy planning: an integrated approach to manage uncertainty, turbulence, and dynamics. Ournal of Knowledge Management, 21(2), 233-253. https://doi.org/10.1108/jkm-02-2016-0071

Buil, I., Martínez, E., \& de Chernatony, L. (2010). Medición del valor de marca desde un enfoque formativo. Cuadernos de Gestion, 10(ESPECIAL), 167-196. https://doi.org/10.5295/ cdg.100204ib 
Burbano, M. J. P., Arciniegas, G., \& Hernández, S. D. R. Á., Chuga, J. F. E. (2019). Factores Que Influyen En Los Emprendimientos De Los Artesanos De San Antonio De Ibarra. Investigación Operacional, 40(2), 242-248. https://doi.org/http://repositorio.espe.edu.ec/handle/21000/14685

Castro Monge, E. (2010). Las estrategias competitivas y su importancia en la buena gestión de las empresas. Ciencias Económicas, 28(1), 247-276.

Chambers, D.(2021, Enero). Líderes Mundiales en Exportaciones de Flores Cortadas. Ripley en Español. https://es.ripleybelieves.com/global-leaders-in-cut-flower-exports-6016

Chirico, F., \& Salvato, C. (2008). Knowledge integration and dynamic organizational adaptation in family firms. Family Business Review, 21(2), 169-181. https://doi.org/10.1111/j.17416248.2008.00117.x

Clark, B.H. (2000). Managerial Perceptions of Marketing Performance: Efficiency, Adaptability, Effectiveness and Satisfaction. Journal of Strategic Marketing, 8(1), 3-25.

Comité de Oxford de Ayuda Contra el Hambre. (s/f) Dónde trabajamos. Kenia. https://www.oxfam. org/es/que-hacemos/dondetrabajamos/paises/kenia

Del Río-Cortina, J. L., Cardona -Arbeláez, D., \& Guacarí-Villalba, A. (2017). Responsabilidad social empresarial y construcción de la marca: una nueva mirada a las estrategias de gestión. Revista De Investigación, Desarrollo E Innovación, 8(1), 49. https://doi.org/10.19053/20278306. v8.n1.2017.7370

Fombrun, C. J., \& Gardberg, N. (2000). Who's Tops in Corporate Reputation? Corporate Reputation Review, 3(1), 13-17. https://doi.org/10.1057/palgrave.crr.1540095

Fraj, E., Matute, J., \& Melero, I. (2015). Environmental strategies and organizational competitiveness in the hotel industry: The role of learning and innovation as determinants of environmental success. Tourism Management, 46, 30-42. https://doi.org/10.1016/j.tourman.2014.05.009

Franco Restrepo, J. G., Restrepo Restrepo, J. C., \& Sánchez Giraldo, J. C. (2014). Marketing management: a competitiveness contribution to small enterprises in the services sector in Medellin, Colombia. Revista Científica Pensamiento y Gestión, 37, 150-174. https://doi.org/10.14482/ pege. 37.7025

Garrido, F.J., Putnam, L.L. (2018) Comunicación Organizacional 2.0 De las metáforas a la pragmática. Comuniteca 2018.

Gaytán Ramírez, M. D. C., \& Flores Villanueva, C. A. (2018). Factores determinantes en la adopción de prácticas de responsabilidad social empresarial: un análisis sectorial en las franquicia. AD-Minister, 33, 21-38. https://doi.org/10.17230/ad-minister.33.2

Gittell, J. H. (2003). The Southwest Airlines. Way, McGraw Hill, New York.

Halim, K. K., Halim, S., \& Felecia. (2019). Business intelligence for designing restaurant marketing strategy: A case study. Procedia Computer Science, 161, 615-622. https://doi.org/10.1016/j. procs.2019.11.164

Hanggraeni, D., Ślusarczyk, B., Sulung, L. A. K., \& Subroto, A. (2019). The Impact of Internal, External and Enterprise Risk Management on the Performance of Micro, Small and Medium Enterprises. Sustainability, 11(7), 2172. https://doi.org/10.3390/su11072172

Hoyos, S., \& Sastoque, J. (2020). Marketing Digital como oportunidad de digitalización de las PYMES en Colombia en tiempo del Covid - 19. Revista Científica Anfibios, 3(1), 39-46. https://doi.org/10.37979/afb.2020v3n1.60 
Jimutai, J. J. (2015) Influence of strategic marketing practices on performance of firms in the flower industry in Kenya [Tesis de maestría] Universidad de Nairobi.

Keatlholetswe, L., \& Malete, L. (2019). Coaching Efficacy, Player Perceptions of Coaches' Leadership Styles, and Team Performance in Premier League Soccer. Research Quarterly for Exercise and Sport, 90(1), 71-79. https://doi.org/10.1080/02701367.2018.1563277

Kimutai, J. J., (2015). Influence of strategic marketing practices on performance of firms in the flower industry in Kenya.

Kotler, P., \& Keller, K. (2006). Marketing Management. Upper Saddle River.

Kotler, P., y Keller, K. L. (2012). Dirección de Marketing (14.a ed.). Pearson Educación.

Kowalkowski, C., Gebauer, H., \& Oliva, R. (2017). Service growth in product firms: Past, present, and future. Industrial Marketing Management, 60, 82-88. https://doi.org/10.1016/j.indmarman.2016.10.015

Marcos Cuevas, J. (2018). The transformation of professional selling: Implications for leading the modern sales organization. Industrial Marketing Management, 69(March 2017), 198-208. https://doi.org/10.1016/j.indmarman.2017.12.017

Martínez Santa María, R., Charterina Abando, J., \& Araujo de la Mata, A. (2010). Un modelo causal de competitividad empresarial planteado desde la VBR: Capacidades directivas, de innovación, marketing y calidad. Investigaciones Europeas de Direccion y Economia de La Empresa, 16(2), 165-188. https://doi.org/10.1016/S1135-2523(12)60117-8

Masoom, M. R., \& Anwar, S. (2013). Top of the Mind Awareness (TOMA) of a particular brand as leading consideration of purchasing the product. An empirical assessment. The Journal of Indian Management \& Strategy, 18(January-March), 13-20.

Milichovsky, F., \& Simberova, I. (2015). Marketing Effectiveness: Metrics for Effective Strategic Marketing.: University of Warwick eResources. 26(2), 211-219. http://0-eds.a.ebscohost.com. pugwash.lib.warwick.ac.uk/eds/pdfviewer/pdfviewer?sid=5fc4b05e-5741-4430-9f05-6b4d294f0eb8\%40sessionmgr4008\&vid=1\&hid=4113

Minciu, M., Berar, F.-A., \& Dobrea, R. C. (2020). New decision systems in the VUCA world. Management \& Marketing. Challenges for the Knowledge Society, 15(2), 236-254. https:// doi.org/10.2478/mmcks-2020-0015

Mizik, N. (2014). Assessing the total financial performance impact of brand equity with limited time-series data. Journal of Marketing Research, 51(6), 691-706. https://doi.org/10.1509/ jmr.13.0431

Mizik, N., \& Jacobson, R. (2008). The financial value impact of perceptual brand attributes. Journal of Marketing Research, 45(1), 15-32. https://doi.org/10.1509/jmkr.45.1.15

Moliner-Velázquez, B., Fuentes-Blasco, M., \& Gil-Saura, I. (2019). Effects of value and innovation on brand equity in retailing. Journal of Brand Management, 26(6), 658-674. https://doi. org/10.1057/s41262-019-00159-5

Mullins, R., Menguc, B., \& Panagopoulos, N. G. (2019). Antecedents and performance outcomes of value-based selling in sales teams: a multilevel, systems theory of motivation perspective. Journal of the Academy of Marketing Science. https://doi.org/10.1007/s11747-019-00705-2

Pacheco, F., \& León Sinche, I. P. (2017). La Asociatividad Del Sector Pesquero Artesanal Del Cantón La Libertad, Provincia De Santa Elena Y La Incidencia En Su Calidad De Vida [Universidad Tecnológica Indoamérica]. http://repositorio.uti.edu.ec//handle/123456789/254 
Park, C. W., MacInnis, D. J., Priester, J., Eisingerich, A. B., \& Iacobucci, D. (2010). Brand attachment and brand attitude strength. Journal of Marketing, 74(6), 1-17.

Peltier, J., Dahl, A. J., \& VanderShee, B. A. (2020). Antecedent consumer factors, consequential branding outcomes and measures of online consumer engagement: current research and future directions. Journal of Research in Interactive Marketing, 14(2), 239-268. https://doi. org/10.1108/JRIM-01-2020-0010

Prawira, Y. (2019). Propuesta de marketing para la fidelización y captación de clientes para la organización MPS Mayorista de Colombia S.A en pantallas industriales LG. In SSRN Electronic Journal (Vol. 5, Issue 564). https://doi.org/10.4324/9781315853178

Quiñónez Gil, P. F. (2013). Análisis de la posibilidad de exportación de flores de otavalo a Corea del Sur para la creación de una campaña publicitaria dirigida a los productores Otavaleños (Bachelor's thesis, Universidad de Guayaquil. Facultad de Comunicación Social. Carrera de Publicidad y Mercadotecnia).

Quist, J., Thissen, W., \& Vergragt, P. J. (2011). The impact and spin-off of participatory backcasting: From vision to niche. Technological Forecasting and Social Change, 78(5), 883-897. https:// doi.org/10.1016/j.techfore.2011.01.011

Ramanathan, R., Ramanathan, U., \& Zhang, Y. (2016). Linking operations, marketing and environmental capabilities and diversification to hotel performance: A data envelopment analysis approach. International Journal of Production Economics, 176, 111-122. https://doi. org/10.1016/j.ijpe.2016.03.010

Reddy, S. K., \& Reinartz, W. (2017). Digital Transformation and Value Creation: Sea Change Ahead. GfK Marketing Intelligence Review, 9(1), 10-17. https://doi.org/10.1515/gfkmir-2017-0002

Rey, C., \& Bastons, M. (2018). Three dimensions of effective mission implementation. Long Range Planning, 51(4), 580-585. https://doi.org/10.1016/j.lrp.2017.07.002

Rizo Rivas, M. (Noviembre 5, 2019). Eficiencia, eficacia, efectividad: ¿son lo mismo?. Forbes México. https://www.forbes.com.mx/eficiencia-eficacia-efectividad-son-lo-mismo/

Romaniuk, J., Wight, S., \& Faulkner, M. (2017). Brand awareness: revisiting an old metric for a new world. Journal of Product and Brand Management, 26(5), 469-476. https://doi.org/10.1108/ JPBM-06-2016-1242

Ruíz, D. (2021). Rentabilidad de marketing y ventas. 08/2021, de Growx Agency Sitio web: https:// www.growxco.com/agency/temas/mejorar-la-rentabilidad-de-marketing-y-ventas

Saritas, O., \& Kuzminov, I. (2017). Global challenges and trends in agriculture: impacts on Russia and possible strategies for adaptation. Foresight, 19(2), 218-250. https://doi.org/10.1108/FS09-2016-0045

Shih, T. Y. (2018). Determinants of enterprises radical innovation and performance: Insights into strategic orientation of cultural and creative enterprises. Sustainability (Switzerland), 10(6). https://doi.org/10.3390/su10061871

Srinivasan, R., \& Lohith, C. P. (2017). Strategic Marketing and Innovation for Indian MSMEs. Strategic Marketing and Innovation for Indian MSMEs, 31-39. https://doi.org/10.1007/978-98110-3590-6

Tokman, M., Mousa, F. T., \& Dickson, P. (2019). The link between SMEs alliance portfolio diversity and top management's entrepreneurial and alliance orientations. International Entrepreneurship and Management Journal, 1001-1022. https://doi.org/10.1007/s11365-019-00597-2 
Tomas, D. (2020). Estrategia de marketing: qué es, tipos y ejemplos. 08/2021, de ONLINE MARKETING \& DIGITAL MARKETING

Torres Huaman, J. N. (2020). Recesión económica y su incidencia en la rentabilidad de empresas del sector hotelero, Santa Anita, 2018-2020. Universidad Cesar Vallejo.

Vera, B. (2007). Apuntes de clases Curso de Comercialización Agropecuaria. Instituto de Economía Agraria. Universidad Austral de Chile. Valdivia. Chile.

Wasike, A.(2020, 28 de marzo) La industria de flores está muriendo debido al COVID-19. Agencia Anadolu. https://www.aa.com.tr/es/mundo/la-industria-de-flores-de-kenia-est $\% \mathrm{C} 3 \% \mathrm{~A} 1$ muriendo-debido-al-covid-19/1782746 